\title{
A Numerical Study on the Flow Characteristics According to the Operating Speed of Hollow Jet Valve
}

\author{
Ki-Hyuk Yang ${ }^{1}$, Jong-Dae Lee ${ }^{2}$, Seung-Won Lee ${ }^{3}$, Hak-Yoon Kim ${ }^{4}$, Dong-jin Sheen ${ }^{5}$, Chang-Hwan Park ${ }^{6 *}$ \\ 1,2,3 Graduate Student, Aeronautical system engineering, Hanseo University, Taean,ChungNam, 32158, Repulic \\ of Korea \\ 4,5,6 Professor,Aeronautical system engineering, Hanseo University, Taean,ChungNam, 32158, Repulic of Korea \\ kihyuk1569@ naver.com ${ }^{1}$,jdlee0818@naver.com², tmddnjs072@naver.com³ \\ heligyro@hanseo.ac.k ${ }^{4}$,viscous@hanseo.ac.kr ${ }^{5}$, lars@hanseo.ac.k ${ }^{6 *}$ \\ Corresponding author*: Dong-jin Sheen : mobile Phone: +82-10-8698-3068
}

Article History: Received:11 november 2020; Accepted: 27 December 2020; Published online: 05 April 2021

Abstract: Various types of valves are installed in the emergency discharge facility of dams, and when water is discharged in an emergency, vibration may occur in the valve depending on operating conditions. In this study, for the hollow jet valve, which is frequently used in the emergency discharge facility of dam, the flow phenomenon occurring according to the operating speed of the valve was observed and the vibration characteristics according to the operating speed were investigated through CFD.In particular, when the hollow jet valve is closed, the valve casing is opened while the inside of the valve casing is filled with air, and the open position is also changed during the opening time, thus the calculation was performed using the unsteady 2-phase overset mesh technique.As a result of calculation through CFD, it was found that when the operating speed of the hollow jet valve was changed, the time range of vibration appeared differently, and the value of the drag coefficient generated was also changed. In addition, it was found that the location of vibration generated according to the operating speed is generated at mostly identical locations, the cause of the vibration is not the vibration caused by the hydraulic pressure exiting the hollow jet valve, and it can be seen that the atmosphere outside the valve flows into the valve casing, creating a complex flow phenomenon inside the valve casing and acting as the cause of vibrating the hollow jet valve.

Keywords: Dam, Hollow Jet Valve, Emergency Discharge, CFD, 2-Phase Flow, Overset Mesh, Fluent

\section{Introduction}

Various types of emergency discharge valves are used in the emergency discharge facility of a dam, and valve types such as cone valves and hollow jet valves are mainly used. The reason for using such a valve is that, as shown in Figure 1, the flow discharged through the valve is distributed around the valve and does not significantly affect the surrounding environment.[1]

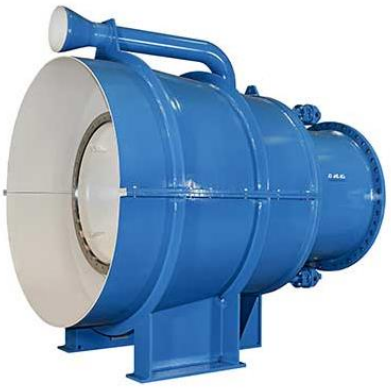

(a) Hollow Jet Valve

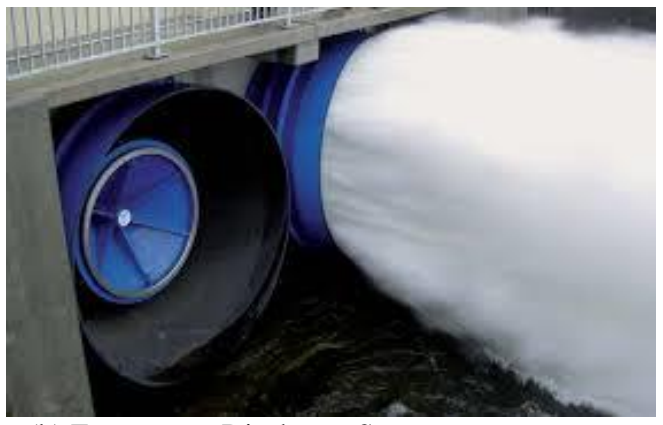

(b) Emergency Discharge State

Figure. 1 Hollow Jet Valve Shape and Emergency Discharge State

The purpose of this paper is to identify the flow characteristics that occur according to the operating speed while the hollow jet valve, among emergency discharge valves, is open, and to acquire basic data to solve the vibration problems that may occur due to this flow phenomenon.

Currently, as a study on the vibration characteristics of Hollow Jet Valve, there is "Hydrodynamic Analysis on Variations of the Hollow-Jet Valve" [2], and this study is a simple comparison of the results of theoretical vibration analysis based on simple momentum theory and simple computational fluid dynamics analysis for inviscid incompressible steady flow under the assumption that the valve is eccentric. In addition to this, computational fluid dynamics analysis for the cavitation generated by the hollow jet valve was performed, but the analysis condition was assumed to be a simple 2 phase flow, and the effect of the cavitation generated at this time on the vibration of the valve was investigated.[3] In addition to this, many studies have been conducted on experiments and computational numerical analysis for hollow jet valve and cone valve, etc. $[4,5,6,7,8,9]$, but there is little research on how the flow phenomenon according to the speed at which the

*Corresponding author: Dong-jin Sheen

Professor,Aeronautical system engineering, Hanseo University, Taean,ChungNam, 32158, Repulic of Korea viscous@hanseo.ac.kr 
hollow jet valve is opened affects the vibration of the valve.

In particular, the method that is widely used as a method of computational flow analysis according to valve opening is to open the valve to an arbitrary position and observe the flow phenomenon while releasing the flow for a certain period of time. However, since the actual valve movement is opened continuously over time while the valve is completely closed, this analysis method cannot simulate the flow phenomenon occurring around the actual valve. Therefore, to simulate the movement of an actual hollow jet valve, computational flow analysis was performed using the overset mesh technique [10].

\section{Computational Methods}

\subsection{Computational Domain}

Figure 2 shows the structure of the hollow jet valve system and is composed of valve, casing, flange, closing chamber, etc. When the flange is moved by hydraulic pressure in the closing chamber, the valve is opened and closed. Computational domain includes a pipe with a length of $17.7 \mathrm{~m}$, and the reason for including the pipe in the computational domain is because the butterfly valve is installed in the front part of the pipe, and to observe whether the vibration by the flow phenomenon is transmitted to the butterfly valve

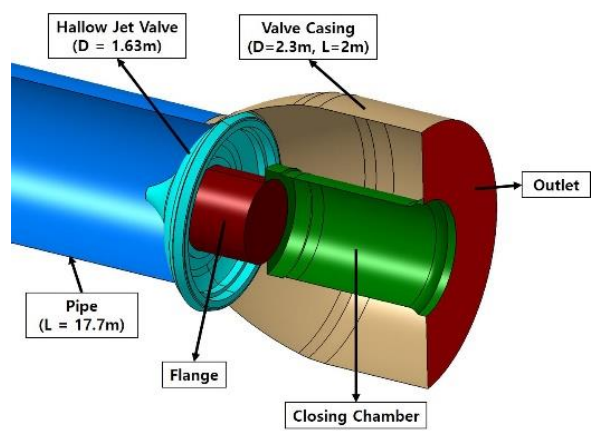

Figure 2. Structure of Hollow Jet Valve

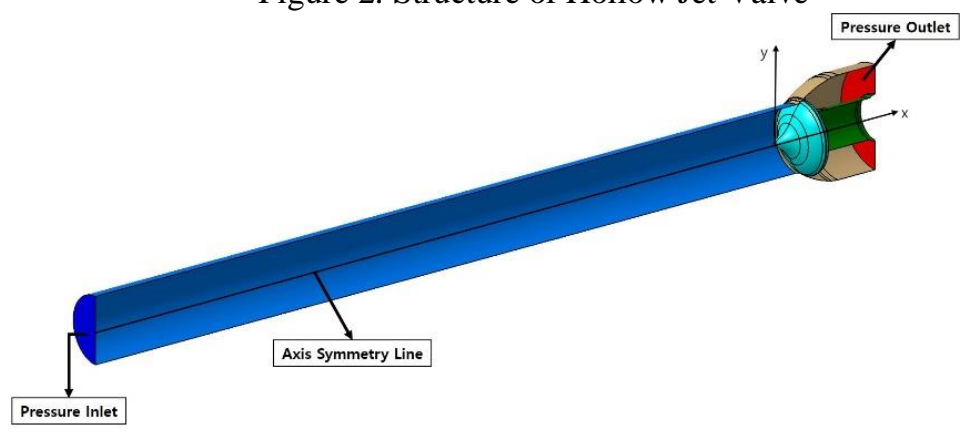

Figure 3. Boundary Condition

\subsection{Boundary Condition}

Figure 3 shows the boundary condition of the computational model. Since the computational domain is in the form of axis symmetry, axis symmetry boundary condition, pressure inlet, and pressure outlet boundary condition were assigned. Head pressure of a constant size of $59.2 \mathrm{~m}$ was applied to the pressure inlet, and atmospheric pressure was applied to the pressure outlet. Especially, when the hollow jet valve is closed, the inside of the valve casing is filled with air, thus it was calculated by giving a two-phase flow boundary condition.

\subsection{Computational Mesh \& Method}

The computational mesh used for the computational flow analysis of the hollow jet valve was the overset mesh. Overset mesh, generally known as chimera mesh, is a mesh system capable of numerically predicting the flow characteristics occurring in the region of interest after constructing several grid systems in which the calculation regions overlap each other.

In general, a calculation grid that is fixed in space is called a background mesh, and a calculation grid that moves on a fixed calculation grid is called a component mesh. In particular, in the case of computational flow analysis in which the valve changes its position over time, such as a hollow jet valve, there is an advantage in that it is possible to easily create a computational mesh while generating a computational mesh of higher quality than a dynamic mesh using a single grid system. 
Figure 4 and Figure 5 show the background mesh created when constructing the overset mesh and the component mesh that moves superimposed on the background mesh, and Figure 6 shows the overset mesh that overlaps the background mesh and the component mesh.

Figure. 4 Background Mesh

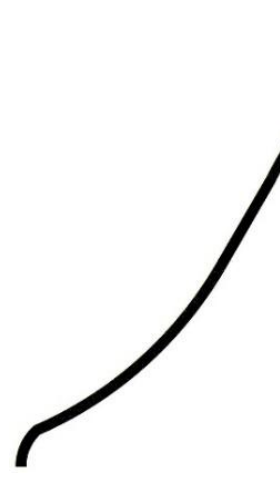

(a) Component Mesh Full View

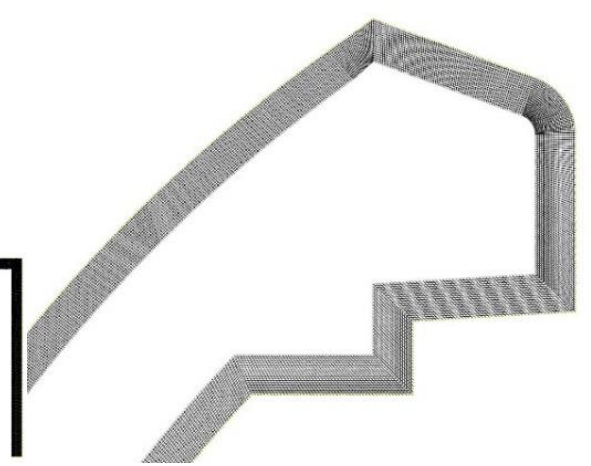

(b) Component Mesh Expanded View

Figure. 5 Component Mesh

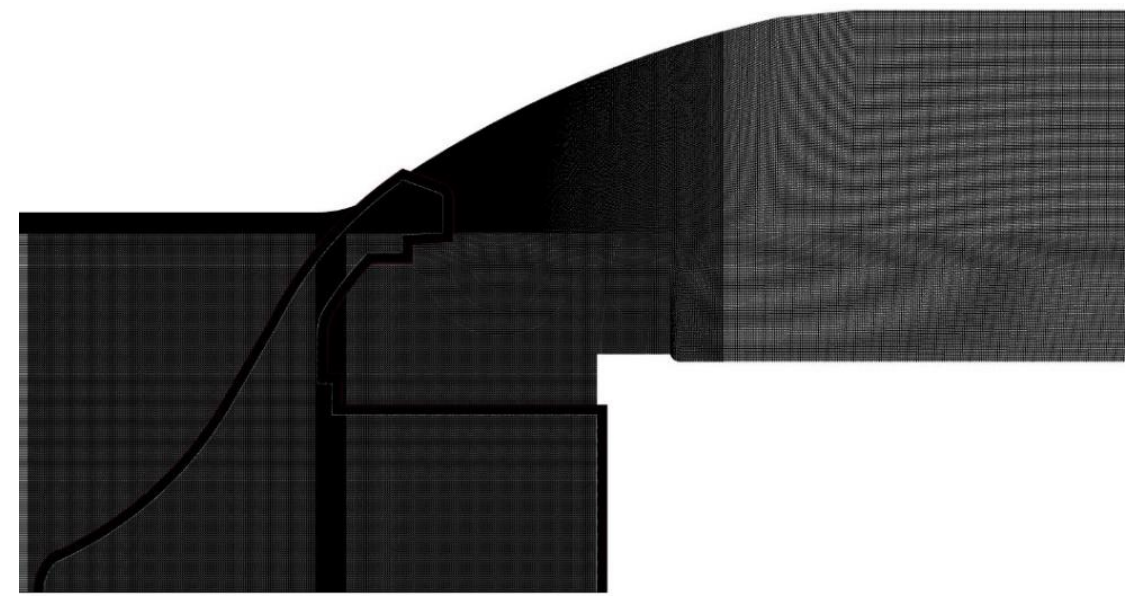

Figure. 6 Overset Mesh

The shape of the mesh used for the unsteady 2-phase calculation is an unstructured rectangular mesh, and the number of meshes is shown in Table 1.

Table 1 : Computational Mesh Size

\begin{tabular}{|c|c|c|c|} 
Mesh & Cells & Faces & Nodes \\
\hline Background & 552,942 & 110,7605 & 554,664 \\
\hline Component & 44,593 & 91,552 & 46,960 \\
\hline Overset & 597,535 & $1,199,157$ & 601,624
\end{tabular}

The commercial program used for computational flow analysis of the hollow jet valve is Ansys Fluent 2019 R3, the analysis technique used is unsteady 2 phase flow, and the turbulence model used for turbulence 
calculation is a $\kappa-\varepsilon$ realizable model. The opening speed of the hollow jet valve installed in the emergency discharge facility was calculated in 3 types at $0.1 \mathrm{~m} / \mathrm{min}$ intervals as shown in Table 2 .

Table 2 : Stroke distance and operating condition

\begin{tabular}{|c|c|c|}
\hline Stroke Distance & Operating Speed & Operating Time \\
\hline \multirow{3}{*}{$470 \mathrm{~mm}$} & $0.1 \mathrm{~m} / \mathrm{min}$ & $282 \mathrm{sec}$ \\
\cline { 2 - 3 } & $0.2 \mathrm{~m} / \mathrm{min}$ & $141 \mathrm{sec}$ \\
\cline { 2 - 3 } & $0.3 \mathrm{~m} / \mathrm{min}$ & $94 \mathrm{sec}$ \\
\hline
\end{tabular}

\section{Results and Discussion}

When the opening speed of the hollow jet valve installed in the emergency discharge facility of a dam is opened as shown in Table 2, the vibration characteristics generated by the flow phenomenon around the hollow jet valve are as shown in Figure 7.

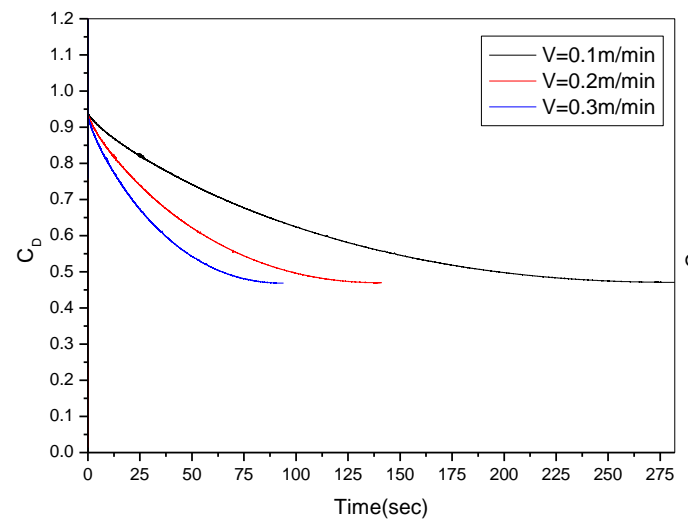

(a) Time $=0 \sim 282 \mathrm{sec}$

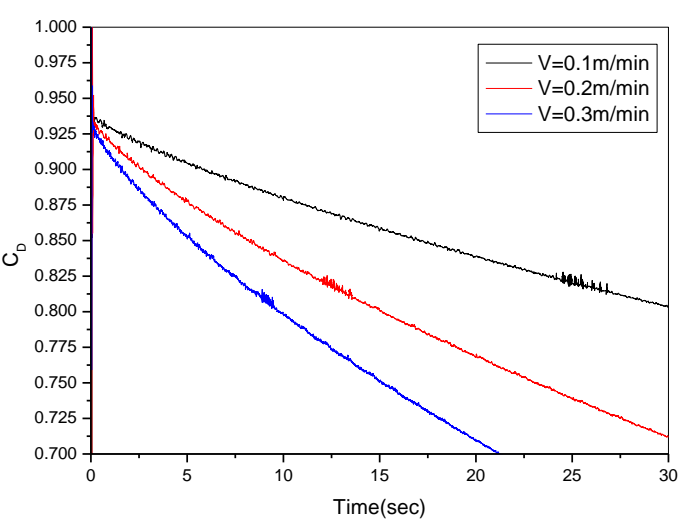

(b) Time $=0 \sim 30 \mathrm{sec}$

Figure 7. Time-Drag Coefficient for Operating Speed

Figure 7 is a graph showing the drag coefficient value generated in the hollow jet valve according to the time change, and the drag coefficient was calculated as Equation (1).

$$
C_{D}=\frac{2 \times \text { Force }}{\rho V^{2} S}
$$

Here, Force is a value calculated from Fluent, $\rho$ is the density of water, $\mathrm{S}$ is the area of the hollow jet valve, and $\mathrm{V}$ is the operating speed of the valve.

In Figure 7 (a), a large increase in the drag coefficient when the valve is opened is a phenomenon that occurs at the beginning of a general valve opening, and it can be seen that the drag coefficient decreases with time and gradually becomes a constant value, and this is because, as shown in Figure 2, the open area of the valve increases and becomes constant over time. In particular, as the operating speed of the hollow jet valve increases, the drag coefficient value decreases significantly, and this is a phenomenon that occurs because the force acting on the hollow jet valve decreases as the operating speed increases because the flow direction of the flow and the operation direction of the valve move in the same direction, and the operating speed of the valve is inversely proportional to the drag coefficient. Looking at Figure 7 (b), when the operating speed of the valve is $0.1 \mathrm{~m} / \mathrm{min}, 0.2 \mathrm{~m} / \mathrm{min}$ and $0.3 \mathrm{~m} / \mathrm{min}$, it can be seen that vibration is generated for about 2.5 seconds, 2 seconds and 1.5 seconds respectively as the operation time passes 27 seconds, 14 seconds, and 8 seconds. The position of the valve corresponding to this time period is between about $40 \mathrm{~mm}$ and $47 \mathrm{~mm}$, and it was found that significant vibration occurs only at this specific position.

Figure $8 \sim$ Figure 10 are figures showing the velocity profile in the region where vibration occurs according to the operating speed. As shown in the figure, it is judged that it is not the vibration caused by the hydraulic pressure exiting the hollow jet valve, but the atmosphere outside the valve flows into the valve casing, creating a complex flow phenomenon inside the valve casing, causing the hollow jet valve to vibrate. 
Ki-Hyuk Yang ${ }^{l}$, Jong-Dae Lee ${ }^{2}$, Seung-Won Lee ${ }^{3}$, Hak-Yoon Kim ${ }^{4}$, Dong-jin Sheen ${ }^{5}$, Chang-Hwan Park ${ }^{6 *}$

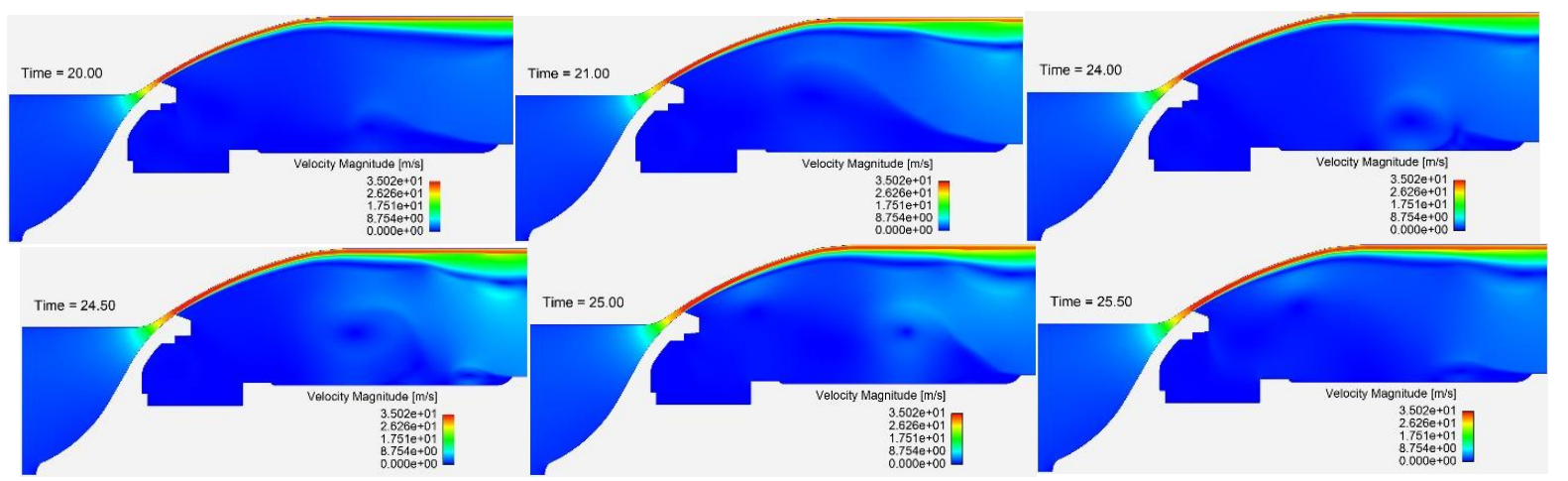

Figure 8. Velocity Profile (Operating Speed $=0.1 \mathrm{~m} / \mathrm{min}$ )

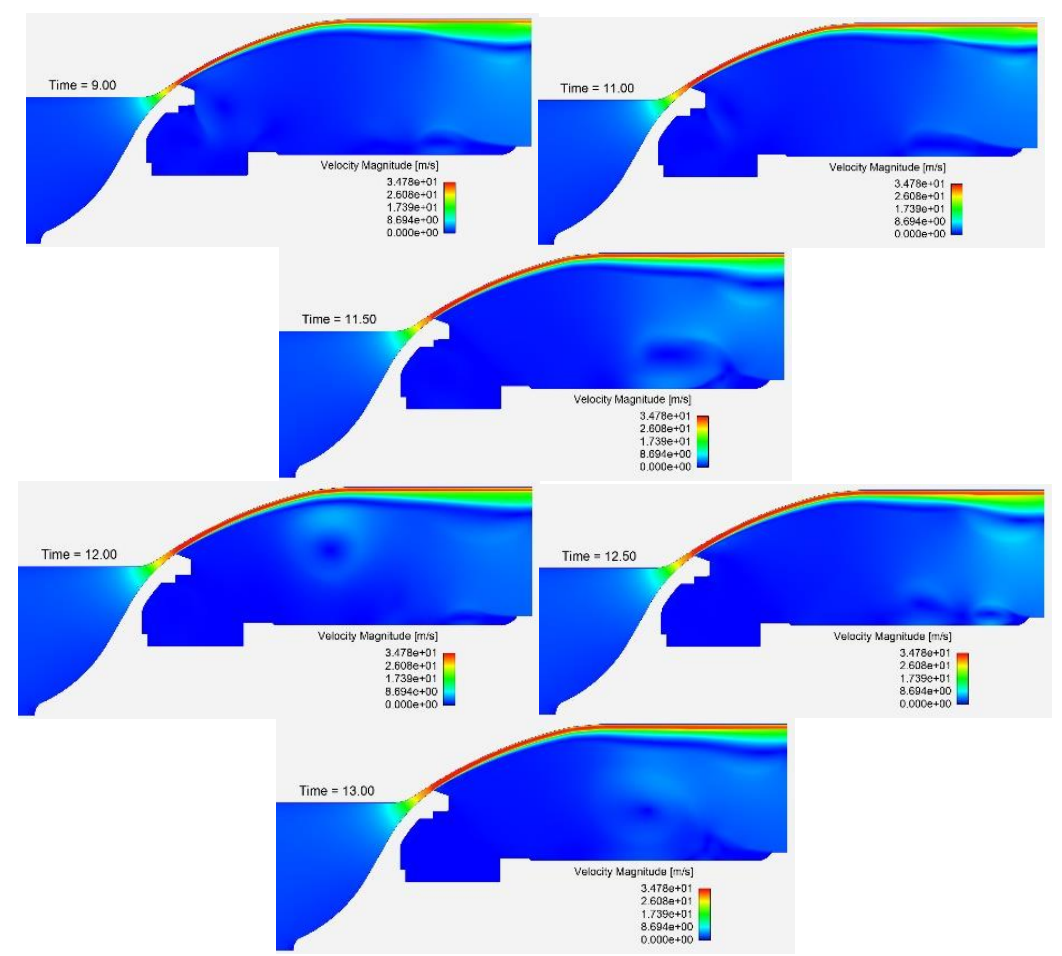

Figure 9. Velocity Profile (Operating Speed $=0.2 \mathrm{~m} / \mathrm{min}$ )

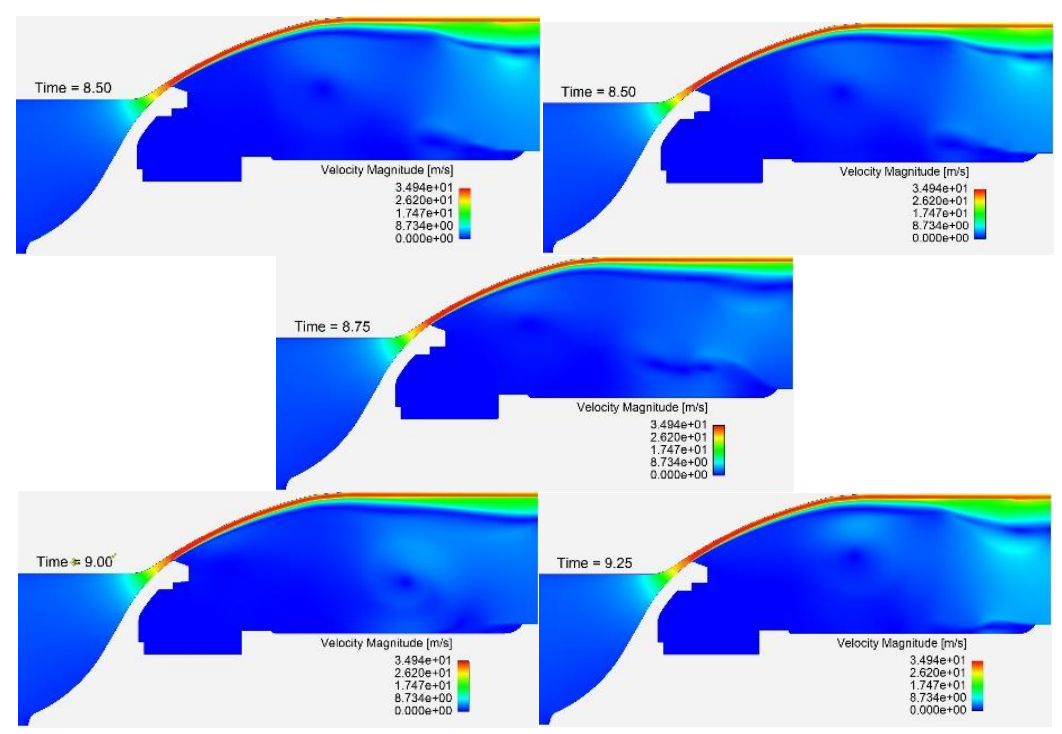




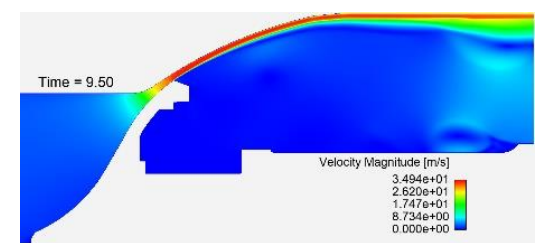

Figure 10. Velocity Profile(Operating Speed $=0.3 \mathrm{~m} / \mathrm{min})$

\section{Conclusion}

Flow characteristics and vibration characteristics were investigated by using the drag coefficient generated from the valve when the operating speed of the hollow jet valve, which is frequently for emergency discharge facility in dams, was varied. It was found that when the operating speed was different, the time range of the vibration was different, and the value of the generated drag coefficient also changed. The difference in drag coefficient depending on the operating speed is a phenomenon that occurs because the operating direction and flow direction of the valve are the same, and the magnitude of the force that appears is different, and the location of vibration generated according to the operating speed was generated at almost the same location. In particular, it is judged that the cause of the vibration is not the vibration caused by the hydraulic pressure exiting the hollow jet valve, but due to the the atmosphere outside the valve flowing into the valve casing, creating a complex flow phenomenon inside the valve casing, causing the hollow jet valve to vibrate.

\section{References}

1. Kouici KURATA \& Toshiaki MAKIHATA (2012). Hydrodynamic Analysis on Vibrations of The Hollow-Jet Valve. The Journal of Japan Society of Civil Engineers, Vol.68, No.4, I_889-I_894.

2. Jiongyang $\mathrm{Wu}(2003)$. Three-Dimensional Simulation of Turbulent Cavitating Flows in a Hollow-Jet Valve. CMES, vol.4 no.6 pp 679-689

3. HW Roh, GS Lee, YM Park, BS Kim, YH Lee(2005). The Study of the Decision Criteria for the Urgency Release Valve in Hydraulic Dam. Korean Society for Fluid Machinery.

4. Commissioner's Offices Denver, Colorado (1960). Calibration of Hollow-Jet Valves and Vibration Studies of Outlet Works Y-Branch Falcon Dam. Hydraulic Laboratory Report No. Hyd-474

5. P.L. Skousen. (2007). Valve Handbook, McGraw-Hill.

6. Barry Jacob Prettyman (2014). Considerations for Hood Placement and Design Downstream from a Fixed-Cone Valve. Utah State University Logan, Utah.

7. Crow, D. A., and Washbourn, J. B. (1985). Investigation into the trajectories of jets issuing from fixed cone valves. Papers presented at the International Conference on Developments in Valves \& Actuators for Fluid Control, Oxford, England.

8. Stephens, D., Johnson, M. C., and Sharp, Z. B. (2012). Design considerations for fixed cone valve with baffled hood. J. of Hydraulic Engrg., 138(2), 204-209.

9. Ji-Won Choi, Sun-Hyung Park, Kwon-Hee Lee.(2016). Flow Analysis and Experimental Study of Globe Valve for Precision Control. Journal of the Korea Academia-Industrial Cooperation Society, Vol. 17, No. 7, pp. 734-739.

10. Ansys Inc., Ansys Fluent Manual 